\title{
Geodara y la lista de localidades y divisiones administrativas de Aragón: dos herramientas eficaces para el control de la información geográfica
}

Geodara and the list of towns and administrative divisions of Aragon: two powerful tools for the control of geographic information

\author{
Marta MASTRAL GASCón DE GOTOR \\ Archivo Histórico Provincial de Huesca (Huesca), mmastral@aragon.es
}

\begin{abstract}
Resumen
Se presentan dos normas vinculadas a la descripción documental en el ámbito archivístico: Geodara, manual que sistematiza la creación de descriptores geográficos y la Lista de localidades, municipios, comarcas y provincias, actuales y desaparecidos de Aragón, ejemplo de la aplicación de Geodara a las denominaciones del territorio aragonés. Se analizan su desarrollo y sus aplicaciones.
\end{abstract}

Palabras clave: Topónimos. Descriptores geográficos. Normalización.

\section{Introducción}

El Grupo de Trabajo de Descriptores Geográficos de Aragón (GDGA) se crea en junio de 2009, impulsado por la Dirección General de Patrimonio Cultural del Gobierno de Aragón, con el objetivo de normalizar los puntos de acceso geográficos que se utilizarán en la descripción de los bienes del patrimonio cultural aragonés -y, en particular, de los fondos depositados en los archivos aragoneses-; así como para recuperar información de ambos sectores a través de los buscadores de SIPCA y de DARA.

En el cumplimiento de sus funciones, el GDGA desarrolló dos líneas de trabajo:

1. Redactar Geodara: Norma aragonesa para la creación de descriptores geográficos (Grupo de Trabajo de Descriptores Geográficos de Aragón, 2011a).

2. Establecer los descriptores geográficos correspondientes a las localidades y divisiones administrativas de Aragón. Concretamente construyó, aplicando Geodara, la Lista de localidades, municipios, comarcas y provincias, actuales y desaparecidos, de Aragón, con las principales relaciones jerárquicas en-

\begin{abstract}
Two standards for archival description are presented: Geodara, a handbook that systematizes the use of geographical descriptors, and the List of the current and disappeared localities, municipalities, districts and provinces of Aragon. This list is an example of the application of Geodara to the toponymy of the region of Aragon. Their development and applications are discussed.
\end{abstract}

Keywords: Place names. Geographic descriptors. Standardization.

tre ellos (Grupo de Trabajo de Descriptores Geográficos de Aragón, 2011b).

\section{La normalización de los nombres geográficos}

La búsqueda en sistemas de información automatizados exige normalizar tanto las descripciones como los puntos de acceso.

Tradicionalmente, los elementos que nos ayudan a extraer el documento adecuado de entre todos los existentes en el universo de búsqueda son los nombres de persona, los de institución, las materias y los lugares en los que se ubica o a los que hace referencia el documento.

Concretamente, la búsqueda por lugar ha sido y es uno de los sistemas de exploración más utilizados por profesionales y usuarios. De hecho, los índices de lugar son instrumentos de descripción fundamentales en la archivística tradicional.

Pero la eficacia de todos estos puntos de acceso es directamente proporcional a su grado de normalización. Por tanto, es necesario normalizar los nombres de lugar para que los resultados de nuestras búsquedas documentales sean exhaustivos, pertinentes y precisos. 
En España no existía una norma específica y suficientemente desarrollada para normalizar los puntos de acceso geográficos. La publicación de Geodara en junio de 2010, vino a solventar esta carencia. En junio de 2011 se publicó una segunda edición corregida y aumentada, que actualmente sigue en vigor.

\section{Trabajos previos a la redacción de la norma}

Para la elaboración de Geodara se trabajó en una doble vía.

Por un lado, se realizó un proceso de investigación bibliográfica (Grupo de Trabajo de Descriptores Geográficos de Aragón, 2011a, p. 78 y 79) que concluyó con el estudio de las normas de descripción archivística y bibliográfica publicadas, las normas sobre confección de lenguajes documentales y los propios lenguajes controlados, con el fin de analizar lo establecido por la normativa respecto a la creación de descriptores de lugar.

Paralelamente, se realizó una consulta a una selección de archivos con el fin de conocer la práctica habitual que se llevaba a cabo en los centros a la hora de incluir topónimos en sus bases de datos.

Con la información obtenida del estudio teórico y las respuestas de los archiveros, llegamos a dos conclusiones fundamentales:

1. La norma tendría que tratar todos los aspectos relativos a los nombres de lugar contemplados en la bibliografía estudiada.

2. La norma tendría que resolver todas las dudas que se planteaban los profesionales en su quehacer diario. Dudas como las siguientes: qué es un lugar (un río, un edificio, un camino, un parque cultural, etc.); cuál es el nombre correcto de un lugar -el más conocido, el que se extrae de los documentos, el que se extrae de la bibliografía, etc.-; qué ocurre cuando un nombre designa varios lugares; qué sucede con los nombres antiguos y con los extranjeros, etc.

Es decir, se definió el alcance de la norma que se va iba a redactar, qué ámbito tenía que cubrir y qué dudas tenía que solventar.

\section{Contenido y estructura de Geodara}

Geodara se redactó teniendo en cuenta estas premisas y con el objetivo de resolver todas las dudas que plantean las denominaciones locales.
La norma parte de un concepto amplio de lugar, que incluye todas las posibles ubicaciones de bienes, personas y acontecimientos.

Siendo el objeto de normalización tan amplio, ha sido necesario subdividirlo en varios tipos de lugares. Esta división origina los cinco apartados en los que se estructura Geodara: A) Divisiones administrativas y localidades; B) Accidentes geográficos; C) Construcciones y yacimientos arqueológicos; D) Zonas geográficas; y E) Delimitaciones rústicas y urbanas.

Para todos y cada uno de los cinco tipos de lugares se indican las fuentes autorizadas donde localizar el nombre de cada sitio, la forma de convertir dicho nombre en un descriptor geográfico, es decir, libre de ambigüedades, y las pautas para su formalización.

Cada uno de los cinco tipos de lugares, repite la misma estructura:

1. Concepto.

2. Elección del nombre: fuentes de información y criterios de selección del nombre.

3. Componentes del descriptor: término de indización, calificadores y atributos.

4. Formalización: orden de las palabras, ortografía, fechas, lengua, grafías, y abreviaturas y siglas.

La norma cuenta, además, con un glosario que define de forma somera los términos utilizados que puedan resultar dudosos, o aquellos que se ha considerando interesante incluir por tener un matiz semántico específico en este contexto.

El resultado es una herramienta con la que se puede construir cualquier descriptor de lugar, de todo tipo, perteneciente a cualquier contexto y época; si bien es cierto que desarrolla con mayor detalle y ejemplifica sobre todo los lugares que, de forma habitual, van unidos a los bienes $\mathrm{y}$ fondos aragoneses.

\section{Pautas en la aplicación de Geodara}

Para una correcta aplicación de Geodara se recomienda seguir la siguiente pauta:

1. En primer lugar, vincular el lugar cuyo nombre hay que normalizar a uno de los cinco tipos establecidos.

2. A continuación seguir los pasos que desarrolla el apartado correspondiente, esto es: a) comprobar si está recogido por la fuente principal; b) establecer los componentes del término de indización; y c) formalizar dichos componentes. 


\section{Principales acuerdos}

Se exponen a continuación algunos de los principales acuerdos que rigen Geodara. Todos ellos han sido estudiados, discutidos, razonados y adoptados de común acuerdo.

1. Qué normaliza Geodara. Geodara regula exclusivamente la creación de descriptores geográficos, pero no es una norma para construir registros de autoridad completos, y tampoco se obtienen de forma automática, tras su aplicación, tesauros de topónimos.

2. Un solo nombre autorizado para cada lugar. Cada lugar tendrá un solo nombre autorizado y cada nombre autorizado hará referencia a un solo lugar. Es una relación unívoca entre el referente y el término que lo nombra.

3. Una sola lengua para cada descriptor. Los términos en otras lenguas se recogerán como términos no autorizados, activando las relaciones de equivalencia.

4. Elección del nombre y fuentes de información. El nombre del lugar es el que recogen las fuentes de información previstas para cada tipo de lugar. Para evitar ambigüedades y dudas, se ha estipulado el orden para su consulta y las normas de aplicación.

5. Nombre antiguo y nombre actual. En el caso de que un mismo lugar haya sido denominado por varios nombres a lo largo de su historia, siendo uno de ellos el actual, se toma como único nombre el actual y los demás pasan a ser términos no aceptados. Esta regla tiene dos excepciones: las carreteras y las delimitaciones rústicas y urbanas.

6. Nombres dudosos o ilegibles. En el caso de nombres de lugares dudosos, ilegibles o de difícil comprensión se propone utilizar un ámbito geográfico más amplio.

7. Lugares sin nombre. Se construirá el descriptor mediante un nombre común que indique de forma genérica el tipo de lugar del que se trata, y tantos calificadores como sea necesario para que el descriptor resulte unívoco.

8. Uso de calificadores. El descriptor de lugar podrá estar formado por el término de indización, el calificador y el atributo. El calificador solo se usará para deshacer homonimias, existentes o probables.

\section{La Lista de localidades, municipios, comarcas y provincias, actuales y desaparecidos, de Aragón}

\subsection{Qué es y qué contiene}

La Lista reúne las denominaciones de las divisiones administrativas (provincias, comarcas y municipios) y de las localidades de Aragón, normalizadas de acuerdo a Geodara.

Comprende todos los lugares de este tipo que existen en la actualidad, así como los municipios y localidades desaparecidos de los que haya quedado constancia documental. El número de términos recogidos en la versión 2.1, correspondiente a julio de 2011, es de 3.260.

Se puede considerar el primer producto resultante de la aplicación de Geodara.

\subsection{Finalidad}

Esta herramienta proporciona al usuario una serie de descriptores de lugar para ubicar de forma correcta y unívoca en el territorio aragonés un documento o un bien cultural.

El uso de estos términos tiene como objetivo final la adecuada indización y la eficaz recuperación de la información en sistemas de búsqueda manuales o automatizados.

\subsection{Confección de la Lista}

\subsubsection{Fuentes de información}

Los términos incluidos proceden de las fuentes de información que a continuación se relacionan:

- Nomenclátor de Aragón por provincias de 2010 (Instituto Aragonés de Estadística, 2011).

- Ubieto Arteta, Antonio (1984). Historia de Aragón: los pueblos y los despoblados.

- Catálogo de pueblos y municipios de Aragón. Estadística de población y nomenclaturas toponímicas entre 1900 y 2004 (Instituto Aragonés de Estadística, 2005).

- Nomenclátores de las ciudades, villas, lugares, aldeas y de demás entidades de población de España (España, 1857-1996). Se han utilizado los nomenclátores correspondientes a los años 1857, 1858, 1900, 1910, 1920, 1930, 1940, 1950, 1970, 1981, 1991 y 1996. 


\subsubsection{Depuración y normalización de los términos}

Las denominaciones procedentes de las fuentes de información, antes de ser incluidas en la lista, tuvieron que ser adaptadas, con los objetivos básicos de depurar posibles duplicados de términos cuya pertinencia resultaba dudosa, y de convertir los nombres de lugar incorporados en términos de indización, según las pautas de la norma.

Las operaciones concretas que se realizaron fueron:

1. Depuración de términos. La tarea de depuración concluyó con la eliminación de cierto número de términos, bien por estar duplicados, bien porque su presencia en el listado se consideró poco pertinente, pues, a pesar de tratarse de lugares habitados, no correspondían con claridad a ninguno de los niveles recogidos en esta lista.
2. Formalización en orden directo. Siguiendo las pautas de Geodara, todos los componentes se formalizarán según un orden basado en el lenguaje natural, suprimiéndose la inversión de elementos en los casos de nombres que incluían artículos.

3. Resolución de homonimias. En el caso de aquellas palabras con varios significados, se ha actuado, también según Geodara, añadiendo un calificador entre paréntesis a cada uno de los términos que presentaran este problema.

4. Resolución de sinonimias. Sólo se detectó un tipo de sinonimia: el de las localidades que han tenido varios nombres sucesivos en el tiempo. En estos casos se determinó, tal y como indica la norma, elegir el nombre actual o, en el caso de tratarse de una localidad desaparecida, el último nombre por el que fue conocida la localidad en cuestión.

\begin{tabular}{|c|c|c|c|c|}
\hline Descriptor & Tipo & Provincia & Comarca & Municipio \\
\hline Ababuj & localidad & Teruel & Comunidad de Teruel & Ababuj \\
\hline Ababuj (municipio) & municipio & Teruel & Comunidad de Teruel & \\
\hline Abad & localidad desaparecida & Zaragoza & Campo de Daroca & Anento \\
\hline Abanto & localidad & Zaragoza & Comunidad de Calatayud & Abanto \\
\hline Abanto (municipio) & municipio & Zaragoza & Comunidad de Calatayud & \\
\hline Abay & localidad & Huesca & La Jacetania & Jaca \\
\hline Abay (municipio desaparecido) & municipio desaparecido & Huesca & La Jacetania & \\
\hline Abefito & localidad desaparecida & Zaragoza & Campo de Daroca & Daroca \\
\hline Abejuela & localidad & Teruel & Gúdar-Javalambre & Abejuela \\
\hline Abejuela (municipio) & municipio & Teruel & Gúdar-Javalambre & \\
\hline Abella & localidad & Huesca & La Ribagorza & Laspaúles \\
\hline Abellada & localidad desaparecida & Huesca & Alto Gállego & Sabiñánigo \\
\hline Abena & localidad & Huesca & La Jacetania & Jaca \\
\hline Abena (municipio desaparecido) & municipio desaparecido & Huesca & La Jacetania & \\
\hline Abenfigo & localidad & Teruel & Maestrazgo & Castellote \\
\hline Abenilla & localidad & Huesca & Alto Gállego & Sabiñánigo \\
\hline Abenozas & localidad & Huesca & La Ribagorza & Graus \\
\hline Abi & localidad & Huesca & La Ribagorza & Seira \\
\hline Abi (municipio desaparecido) & municipio desaparecido & Huesca & La Ribagorza & \\
\hline Abiego & localidad & Huesca & Somontano de Barbastro & Abiego \\
\hline Abiego (municipio) & municipio & Huesca & Somontano de Barbastro & \\
\hline Abizanda & localidad & Huesca & Sobrarbe & Abizanda \\
\hline Abizanda (municipio) & municipio & Huesca & Sobrarbe & \\
\hline Abós & localidad desaparecida & Huesca & La Jacetania & Jaca \\
\hline Abuhán & localidad desaparecida & Teruel & Comunidad de Teruel & Teruel \\
\hline Acered & localidad & Zaragoza & Campo de Daroca & Acered \\
\hline Acered (municipio) & municipio & Zaragoza & Campo de Daroca & \\
\hline
\end{tabular}

Figura 1. Detalle de la Lista

\subsection{Estructura y presentación}

Los descriptores se presentan en forma alfabética, dispuestos en una tabla con varias columnas. De cada descriptor, se suministra la siguiente información: tipo, provincia, comarca y municipio al que se pertenece (Figura 1).

\subsection{Acceso y uso}

La Lista se publica en formato electrónico, accesible tanto en el portal web del Gobierno de Aragón, como en diversos portales especializados dependientes de la Dirección General de Patrimonio Cultural y otros organismos oficiales. 
Se presenta en dos versiones:

- Documentos de texto, en formato PDF.

- Fichero de datos en formato CSV, que permite diversos grados de manipulación, modificación, exportación, etc. A partir de esta versión, el contenido de esta lista es susceptible de ser integrado de diversos modos en las aplicaciones de gestión documental de las que disponga cualquier centro.

\subsection{Actualización y corrección de la Lista}

La Lista no es un instrumento definitivo y cerrado, sino que está abierta a modificaciones. Por un lado, se contemplarán las posibles correcciones y sugerencias aportadas por la comunidad profesional. Por otro, la realidad toponímica también puede cambiar, bien por incorporación de nuevos términos, bien por modificación de los existentes, y con estos cambios se irá renovando y actualizando esta herramienta.

\section{Aplicación de Geodara y de la Lista}

Ni Geodara ni la Lista de descriptores se concibieron como meros trabajos teóricos. Desde el momento de su publicación, se procuró que fuesen instrumentos útiles y prácticos; y se procedió a su inmediata aplicación. Para ello, se buscó un universo de términos suficientemente amplio y variado, que sirviese de banco de pruebas, y se planteó una aplicación sistemática de la norma.

Se utilizaron las bases de datos de descripción de dieciséis archivos aragoneses -los accesibles en ese momento a través de DARA, el buscador de documentos y archivos de Aragón-. Los términos incluidos en el campo lugar se volcaron en una tabla, y se procedió a normalizarlos de acuerdo a Geodara.

En el caso de las localidades y divisiones administrativas recogidas en la Lista, el trabajo consistía simplemente en trasladar el descriptor ya normalizado a la tabla. En el resto de los casos -localidades y divisiones administrativas no aragonesas, así como accidentes geográficos, construcciones y yacimientos arqueológicos, zonas geográficas y delimitaciones rústicas y urbanas- se aplicaron, caso por caso, las directrices de Geodara para construir y formalizar los diferentes tipos de lugares.

El resultado son más de 10.000 términos normalizados, de todos los tipos y épocas.

Es cierto que la práctica sirvió para constatar lo que ya presuponíamos: que la norma hay que interpretarla. Es decir, que hay cuestiones que tiene que decidir la persona responsable de la descripción, y no puede ni le corresponde resolverlas a Geodara. Son cuestiones como: determinar si una entidad es o no es un lugar; distinguir entre lo que es la simple evolución del nombre de un término y lo que son dos términos que aluden a distintos referentes; estipular lo que tiene que ser punto de acceso de una descripción y lo que no tiene que serlo; o determinar qué lugares tienen que incluirse en el campo de una base de datos destinado a los topónimos normalizados frente a lo que tiene que hacerse constar simplemente como palabra clave.

\section{Conclusiones y proyectos de futuro}

Geodara fue la primera norma publicada en España, en el ámbito archivístico, reguladora de la denominación de los nombres de lugar, y está prevista para normalizar cualquier tipo de lugar de cualquier época.

Su aplicación en descripciones reales, procedentes de los archivos aragoneses, ha resultado altamente satisfactoria. No se ha dado, de entre los más de 10.000 términos normalizados, ningún caso al que la norma no pudiese dar solución.

Bien es cierto que la interpretación y la aplicación correcta de Geodara corresponden al profesional, quién tendrá que tomar decisiones que pertenecen más a la teoría general de la descripción y de los lenguajes documentales, que a la normalización de términos concretos.

En todo caso, no es una herramienta estática. Las observaciones procedentes de la práctica irán dando las pautas para su mejora.

Pero, además, la lista de descriptores podrá ir completándose, añadiendo relaciones de equivalencia y asociativas entre sus términos, hasta llegar a convertirse en un tesauro geográfico aragonés.

Un tesauro ilustrado - pues a cada descriptor se le podrá vincular su imagen-, codificado mediante un leguaje de etiquetas, y también georreferenciado, es decir, que ofrezca la posibilidad de buscar los lugares a través de puntos definidos en un mapa mediante coordenadas espaciales.

Todo este trabajo tiene una única finalidad: mejorar el resultado de las búsquedas de datos y documentos en archivos y otros custodios de fondos documentales.

Sin duda, mejorando la calidad de las descripciones y de los puntos de acceso, se mejora la 
cantidad, pertinencia y precisión de los resultados obtenidos.

\section{Referencias}

Grupo de Trabajo de Descriptores Geográficos de Aragón (2011a). Geodara: Norma aragonesa para la creación de descriptores geográficos. Zaragoza: Departamento de Educación, Cultura y Deporte, 2011. http://www.dehuesca.es/ sipca/IMAGEN/documentos w eb/GDGA-manual_geodara_v2.pdf

Grupo de Trabajo de Descriptores Geográficos de Aragón (2011b). Lista de localidades, municipios, comarcas y provincias, actuales y desaparecidos, de Aragón. Zaragoza: Departamento de Educación, Cultura y Deporte,

Fecha de envío: 2012-06-11

Fecha de aceptación: 2012-08-21.
2011. http://www dehuesca es/ sipca/IMAGEN/documen tos_web/GDGA-lista\%20de\%20descriptores_2.1.pdf

Instituto Aragonés de Estadística (2005). Catálogo de pueblos y municipios de Aragón: estadística de población y nomenclaturas toponímicas entre 1900 y 2004 . Zaragoza: Gobierno de Aragón y Caja Inmaculada, 2005.

Instituto Aragonés de Estadística (2011). Nomenclátor de Aragón por provincias. Año 2010. Zaragoza: Instituto Aragonés de Estadística, 2011. http://www.aragon.es (2011-04-29)

Ubieto Arteta, Antonio (1984). Historia de Aragón: los pueblos y los despoblados. Zaragoza: Anúbar, 1984

España (1857-1996). [Nomenclátor de las ciudades, villas, lugares, aldeas y de demás entidades de población de España]. Madrid: Instituto Nacional de Estadística. 